\title{
Upper Estimate of Concentration and Thin Dimensions of Measures
}

by

\author{
H. GACKI, A. LASOTA and J. MYJAK
}

The idea of this article originated during the last visit of Professor Andrzej Lasota in L'Aquila, December 2005. Unfortunately, his untimely death made a common conclusion of our nice discussions impossible. We have completed this paper as a token of respect for our Master and Friend.

Summary. We show upper estimates of the concentration and thin dimensions of measures invariant with respect to families of transformations. These estimates are proved under the assumption that the transformations have a squeezing property which is more general than the Lipschitz condition. These results are in the spirit of a paper by A. Lasota and J. Traple [Chaos Solitons Fractals 28 (2006)] and generalize the classical Moran formula.

1. Introduction. The concept of dimension of sets and measures is a basic tool in diverse branches of mathematics. For example, it is an important characteristic of attractors generated by iterated function systems. Various notions of dimension have been proposed: Hausdorff dimension, fractal dimension, correlation dimension, information dimension, capacity, entropy. These concepts have been widely investigated and used, but unfortunately, all of them are rather hard to calculate.

Recently two other concepts of dimension have been proposed by A. Lasota: the concentration dimension and the thin dimension. These dimensions are often easier to calculate and provide a natural and intrinsic estimation of the Hausdorff dimension and the fractal dimension of sets (see [13], [11]). The concentration dimension is defined by using the Lévy concentration function

2010 Mathematics Subject Classification: Primary 37C45; Secondary 28A80, 11K55, $37 \mathrm{C} 40$.

Key words and phrases: upper concentration dimension, upper thin dimension, squeezing property, invariant measure, iterated function system. 
and it is strongly related to the Hausdorff dimension. The thin dimension is based on the notion of the thin function, which is a kind of anti-concentration function. This dimension is related to the fractal dimension.

Since the calculation of dimensions is rather difficult, it is important to find their estimates. Undoubtedly the most elegant and popular such result is the so called Moran formula (see [16]). Suppose that we have an IFS $\left\{w_{i}: i=1, \ldots, N\right\}$, where all functions $w_{i}$ are strictly contractive with Lipschitz constants $L_{i}$. It is well known that such a system admits an attractor $K$. Then the Hausdorff dimension of $K$ is less than or equal to $d$, where $d$ is the unique solution of the Moran equation

$$
\sum_{i=1}^{n} L_{i}^{d}=1 .
$$

If all $w_{i}$ are similarities with scaling factor $L_{i}$, then the Hausdorff dimension of $K$ is equal to $d$. The above result has been generalized in various directions.

We will find an upper estimate of the concentration dimension of a measure invariant with respect to an iterated function system with a squeezing property, and an upper estimate of the thin dimension of a measure invariant with respect to an iterated function system with condensation.

2. Notation and auxiliary results. Let $(X, \rho)$ be a separable metric space. By $B(x, r)$ (resp. $\left.B^{\circ}(x, r)\right)$ we denote the closed (resp. open) ball with center at $x \in X$ and radius $r>0$. For $A \subset X$ and $x \in X, \bar{A}$ stands for the closure of $A, \operatorname{diam} A$ for the diameter of $A, \partial A$ for the boundary of $A$ and $\rho(x, A)$ for the distance from $x$ to $A$. Occasionally we write $|A|$ in place of $\operatorname{diam} A$. As usual, we denote by $\mathbb{R}, \mathbb{R}_{+}$and $\mathbb{N}$ the sets of reals, of all nonnegative reals and of all positive integers respectively.

By $\mathcal{B}(X)$ we denote the $\sigma$-algebra of Borel subsets of $X$ and by $\mathcal{M}(X)$ the family of all finite Borel measures on $X$. Finally, $\mathcal{M}_{1}(X)$ denotes the space of all measures $\mu \in \mathcal{M}(X)$ such that $\mu(X)=1$.

For $\mu \in \mathcal{M}(X)$ the support of $\mu$ is defined by

$$
\operatorname{supp} \mu=\{x \in X: \mu(B(x, \varepsilon))>0 \text { for every } \varepsilon>0\} .
$$

It is easy to verify that for every fixed $\varepsilon>0$ the function $X \ni x \mapsto$ $\mu\left(B^{\circ}(x, \varepsilon)\right)$ is lower semicontinuous. Moreover, if $(X, \rho)$ is separable, then $\sup \left\{\mu\left(B^{\circ}(x, \varepsilon)\right): x \in X\right\}>0$.

Let $\left\{A_{n}\right\}_{n \in \mathbb{N}}$ be a sequence of subsets of $X$. The lower limit $\mathrm{Li} A_{n}$ and the upper limit Ls $A_{n}$ are defined by the following conditions: A point $x$ belongs to $\operatorname{Li} A_{n}$ if there is a sequence $\left\{x_{n}\right\}$ converging to $x$ such that $x_{n} \in A_{n}$, while $x$ belongs to Ls $A_{n}$ if there is a sequence $\left\{x_{n_{k}}\right\}$ converging to $x$ such that $x_{n_{k}} \in A_{n_{k}}$ for $k \in \mathbb{N}$. If $\operatorname{Li} A_{n}=\operatorname{Ls} A_{n}$, we denote this limit set by $\operatorname{Lt} A_{n}$ and call it the topological or Kuratowski limit of $\left\{A_{n}\right\}$. 
By an iterated function system (briefly IFS) we mean a family of continuous functions

$$
w_{i}: X \rightarrow X, \quad i \in I,
$$

where $I=\{1, \ldots, N\}$.

Given an IFS $\left\{w_{i}: i \in I\right\}$ we define

$$
F(A)=\overline{\bigcup_{i \in I} w_{i}(A)} \quad \text { for } A \subset X .
$$

A closed set $A_{0}$ such that $F\left(A_{0}\right)=A_{0}$ is called invariant with respect to the IFS $\left\{w_{i}: i \in I\right\}$.

If there exists a closed set $A_{0}$ such that $\operatorname{Lt} F^{n}(A)=A_{0}$ for every nonempty bounded subset $A$ of $X$, then the IFS $\left\{w_{i}: i \in I\right\}$ is called asymptotically stable. The set $A_{0}$ is uniquely defined and it is called the attractor or fractal (in the sense of Barnsley). Observe that in the case when $X$ is a compact space, the topological limit coincides with the Hausdorff limit. Note also that the function $F$, in general, is not continuous with respect to the topological limit.

It is well known that if all $w_{i}$ are strictly contractive, then there exists a unique compact set $K$ such that

$$
F(K)=\bigcup_{i=1}^{N} w_{i}(K) .
$$

Moreover, for every compact set $A \subset X, F^{n}(A) \rightarrow K$ in the Hausdorff distance. In the classical theory of iterated function systems the set $K$ is called the attractor or fractal corresponding to the IFS $\left\{w_{i}: i \in I\right\}$ (see [1]). If all $w_{i}$ are lipschitzian function with Lipschitz constant $L_{i}$, then $\operatorname{dim}_{H} K \leq$ $d$, where $d$ is the unique solution of the Moran equation (1). If all $w_{i}$ are similarities with scaling factor $L_{i}$, then $\operatorname{dim}_{H} K=d$.

We say that an IFS $\left\{w_{i}: i \in I\right\}$ is regular if there exists a nonempty subset $I_{0} \subset I$ such that the IFS $\left\{w_{i}: i \in I_{0}\right\}$ is asymptotically stable. The attractor of the subsystem $\left\{w_{i}: i \in I_{0}\right\}$ is called a nucleus of the system $\left\{w_{i}: i \in I\right\}$.

Let $\left\{w_{i}: i \in I\right\}$ be a regular IFS and let $A_{0}$ be a nucleus of this system. Define

$$
A_{*}=\overline{\bigcup_{i \in I} F^{n}\left(A_{0}\right)},
$$

where $F$ is given by (2).

Proposition 1 ([12]). Let $\left\{w_{i}: i \in I\right\}$ be a regular IFS and let $A_{*}$ be the corresponding semifractal defined by (3). Then: 
(i) $A_{*}$ does not depend on the choice of nucleus;

(ii) $A_{*}$ is the smallest set invariant with respect to IFS;

(iii) $\operatorname{Lt} F^{n}(A)=A_{*}$ for every nonempty subset $A$ of $A_{*}$.

An operator $P: \mathcal{M}_{1} \rightarrow \mathcal{M}_{1}$ is called Markov if:

- $P\left(\lambda_{1} \mu_{1}+\lambda_{2} \mu_{2}\right)=\lambda_{1} P \mu_{1}+\lambda_{2} P \mu_{2}$ for $\lambda_{1}, \lambda_{2} \in \mathbb{R}_{+}, \mu_{1}, \mu_{2} \in \mathcal{M}_{1}$,

- $P \mu(X)=\mu(X)$.

A measure $\mu^{*} \in \mathcal{M}_{1}$ is called invariant with respect to $P$ if $P \mu_{*}=\mu_{*}$. The operator $P$ is called asymtotically stable if it admits an invariant measure $\mu_{*}$ and $\left\{P^{n} \mu\right\}$ converges weakly to $\mu_{*}$ (i.e $\int_{X} f d P^{n} \mu \rightarrow \int_{X} f d \mu$ for every continuous function $f: X \rightarrow \mathbb{R})$.

The family $\left\{\left(w_{i}, p_{i}\right): i \in I\right\}$, where $w_{i}: X \rightarrow X, p_{i}: X \rightarrow(0,1), i \in I$, are continuous functions and $\sum_{i \in I} p_{i}(x)=1$ for all $x \in X$, is called an IFS with probabilities.

Given an IFS $\left\{\left(w_{i}, p_{i}\right): i \in I\right\}$ we can define a Markov operator $P$ : $\mathcal{M}_{1} \rightarrow \mathcal{M}_{1}$ by

$$
P \mu(A)=\sum_{i \in I} \int_{w_{i}^{-1}(A)} p_{i}(x) \mu(d x), \quad A \in \mathcal{B}(X) .
$$

We say that a measure $\mu$ is invariant with respect to the $\operatorname{IFS}\left\{\left(w_{i}, p_{i}\right)\right.$ : $i \in I\}$ if it is invariant with respect to the corresponding Markov operator. Similarly, IFS is called asymptotically stable if $P$ is asymptotically stable. In particular, if all functions $p_{i}$ are constant the invariant measure satisfies the condition

$$
\mu(A)=\sum_{i \in I} p_{i} \mu\left(w_{i}^{-1}(A)\right), \quad A \in \mathcal{B}(X) .
$$

It is well known that if all functions $w_{i}$ are lipschitzian with Lipschitz constants $L_{i}$ the probabilities $p_{i}$ are constant, and if

$$
\sum_{i \in I} p_{i} L_{i}<1
$$

then the IFS $\left\{\left(w_{i}, p_{i}\right): i \in I\right\}$ is asymptotically stable.

Proposition 2 ([12]). Assume that the IFS $\left\{\left(w_{i}, p_{i}\right): i \in I\right\}$ is asymptotically stable and the IFS $\left\{w_{i}: i \in I\right\}$ is regular. Moreover, assume that $p_{i}(x)>0$ for $x \in X$ and $i \in I$. Then

$$
A_{*}=\operatorname{supp} \mu_{*},
$$

where $A_{*}$ is a semifractal of the IFS $\left\{w_{i}: i \in I\right\}$ and $\mu_{*}$ is an invariant measure with respect to the IFS $\left\{\left(w_{i}, p_{i}\right): i \in I\right\}$.

In the theory of iterated function systems normally the functions under consideration are supposed to be contractions or more generally lipschitzian. 
Here we assume that they have the so called squeezing property. This property has been frequently used in the theory of differential equations (see $[2]-[10],[15]-[17])$ and more recently in the theory of iterated function systems (see $[7,8,9,14,19,20]$ ).

Squeezing Property. Let $A$ be a nonempty bounded subset of $X$. We say that a family of functions $\left\{w_{i}: i \in I\right\}, w_{i}: A \rightarrow A$, has the squeezing property if there exist nonexpansive mappings $P_{i}: A \rightarrow \mathbb{R}^{k_{i}}$ and constants $L_{i} \in(0,1)$ and $c_{i} \geq 0$ for $i \in I$ such that

$$
\rho\left(w_{i}(x), w_{i}(y)\right) \leq \max \left\{L_{i} \rho(x, y), c_{i}\left\|P_{i}(x)-P_{i}(y)\right\|_{i}\right\} \quad \text { for } x, y \in X,
$$

where $\|\cdot\|_{i}$ denotes the norm in $\mathbb{R}^{k_{i}}$.

The following covering property of Euclidean spaces is essential for further results:

Covering Property. Let $L \in(0,1), c>0$ and $\left(\mathbb{R}^{k},\|\cdot\|\right)$ be given. Then there exists an integer $m \geq 1$ such that for every set $B \subset \mathbb{R}^{k}$ with $\operatorname{diam} B \leq c$ there exist Borel sets $\Delta_{1}, \ldots, \Delta_{m}$ such that

$$
B \subset \bigcup_{j=1}^{m} \Delta_{j} \quad \text { and } \quad\left|\Delta_{j}\right| \leq L \quad \text { for } j=1, \ldots, m .
$$

Furthermore, in the case when $\operatorname{diam} B \leq c r$ we can find sets $\Delta_{j}, j=$ $1, \ldots, m$, such that

$$
B \subset \bigcup_{j=1}^{m} \Delta_{j} \quad \text { and } \quad\left|\Delta_{j}\right| \leq L r \quad \text { for } j=1, \ldots, m .
$$

Lemma 1. Let $X$ be a metric space. Suppose that a family $\left\{w_{i}: i \in I\right\}$ has the squeezing property. Let $r>0$. For every $i \in\{1, \ldots, N\}$ there exists $m_{i}$ (chosen according to the Covering Property) such that for every $B \subset X$ with $|B| \leq r$ there are sets $D_{1}^{i}, \ldots, D_{m_{i}}^{i}$ such that

$$
w_{i}(B) \subset \bigcup_{j=1}^{m_{i}} D_{j}^{i} \quad \text { and } \quad\left|D_{j}^{i}\right|<L_{i} r \quad \text { for } j=1, \ldots, m_{i} .
$$

Proof. The proof is implicitly contained in the first step of the proof of Theorem 2.1 in [14]. For the convenience of the reader we give the main idea, thus making our exposition self-contained. For $i \in\{1, \ldots, N\}$ let $m_{i}$ be the integer chosen according to the Covering Property and related to the constants $c_{i}, L_{i}$ and the space $\mathbb{R}^{k_{i}}$. Let $B$ be a Borel set such that $|B| \leq r$. Recall that $P_{i}(B) \subset \mathbb{R}^{k_{i}}$. Since $\left|c_{i} P_{i}(B)\right| \leq c_{i} r$, by the Covering Property there exist sets $\left\{\Delta_{1}, \ldots, \Delta_{m_{i}}\right\}$ such that $c_{i} P_{i}(B) \subset \bigcup_{j=1}^{m_{i}} \Delta_{j}$ and $\left|\Delta_{j}\right| \leq r L_{i}$ for $j=1, \ldots, m_{i}$. Define

$$
D_{j}^{i}=w_{i}^{-1}\left(\left(c_{i} P_{i}\right)^{-1}\left(\Delta_{j}\right) \cap B\right), \quad j=1, \ldots, m_{i} .
$$

Simple calculation shows that such sets satisfy condition (6). 
Lemma 2. Let $\alpha_{i}, \beta_{i}, L_{i} \in(0,1)$ for $i \in J$ be given, where $J$ is an arbitrary set of indices. Let $\mu$ be a probability measure and let $\Phi: \mathbb{R}_{+} \rightarrow \mathbb{R}_{+}$be a bounded increasing function. Suppose that for some $a>0$ the function $\Phi$ satisfies the following condition:

$$
\Phi(r) \geq \sup _{i \in J} \alpha_{i} \Phi\left(r / L_{i}\right) \quad \text { for } r \in(0, a) .
$$

Then there exists $c>0$ such that

$$
\Phi(r) \geq c r^{s} \quad \text { for } r \in(0, a),
$$

where

$$
s=\min _{i \in J} \frac{\log \alpha_{i}}{\log L_{i}}
$$

For the proof of this lemma we refer to [13].

Lemma 3. Let $L_{i}, p_{i} \in(0,1)$ for $i \in 1, \ldots, N$ and $\alpha>0$ be given. Let functions $\varphi, \psi:(0, \infty) \rightarrow[0, \infty)$ be such that

$$
\begin{array}{ll}
\psi(r) \geq \min \left\{\alpha, p_{1} \psi\left(r / L_{1}\right), \ldots, p_{N} \psi\left(r / L_{N}\right)\right\} & \text { for } 0<r \leq r_{0}, \\
\varphi(r) \leq \min \left\{\alpha, p_{1} \varphi\left(r / L_{1}\right), \ldots, p_{N} \varphi\left(r / L_{N}\right)\right\} & \text { for } 0<r \leq r_{0} .
\end{array}
$$

Suppose that

$$
\varphi(r) \leq \psi(r)
$$

for $r \in\left[l r_{0}, r_{0}\right]$, where $l=\min \left\{L_{1}, \ldots, L_{N}\right\}$. Then inequality (9) holds for every $r \in\left(0, r_{0}\right]$.

Proof. Let $L=\max \left\{L_{1}, \ldots, L_{N}\right\}$. Using an induction argument one can show that for every $n \in \mathbb{N}$, inequality (9) holds for $r \in\left[L^{n+1} l r_{0}, r_{0}\right]$. Since $L^{n+1} \rightarrow 0$ as $n \rightarrow \infty$, the statement follows.

3. Upper bound for upper concentration dimension. Given a Borel measure $\mu \in \mathcal{M}_{1}$ the upper concentration dimension of $\mu$ is given by the formula

$$
\overline{\operatorname{dim}}_{L} \mu=\limsup _{r \rightarrow 0} \frac{\log Q_{\mu}(r)}{\log r}
$$

where

$$
Q_{\mu}(r)=\sup \{\mu(A): A \in \mathcal{B}(X),|A| \leq r\} \quad \text { for } r>0
$$

is the well known Lévy concentration function. The values of $Q_{\mu}$ are always positive (see [11, Remark 2.1]).

Theorem 1. Assume that a system $\left\{\left(w_{i}, p_{i}\right): i \in I\right\}$ has the Squeezing Property. Let $\mu$ be an invariant measure with respect to this system. Further, for each couple of numbers $L_{i}, c_{i}, i \in I$, let the integer $m_{i}$ be chosen according 
to the Covering Property. Then

$$
\overline{\operatorname{dim}}_{L} \mu \leq \min _{i \in I} \frac{\log \left(p_{i} / m_{i}\right)}{\log L_{i}} .
$$

Proof. Denote by $\tilde{\mu}$ the outer measure generated by $\mu$, i.e.

$$
\tilde{\mu}(E)=\inf \{\mu(A): A \in \mathcal{B}(X), E \subset A\} \quad \text { for } E \subset X .
$$

Using the relation (4) it is easy to verify that

$$
\tilde{\mu}(E) \geq \sum_{i \in I} p_{i} \tilde{\mu}\left(w_{i}^{-1}(E)\right), \quad E \subset X .
$$

Fix $r_{0}>0$ and let $r \in\left(0, r_{0}\right]$. Let $B \in \mathcal{B}(X)$ be an arbitrary set such that $|B|<r$. From the above inequality and the inclusion $w_{i}^{-1}\left(w_{i}(B)\right) \supseteq B$ it follows that

$$
\tilde{\mu}\left(w_{i}(B)\right) \geq p_{i} \tilde{\mu}\left(w_{i}^{-1}\left(w_{i}(B)\right)\right) \geq p_{i} \mu(B) .
$$

Let $D_{1}^{i}, \ldots, D_{m_{i}}^{i}$ be given by Lemma 1 . Obviously

$$
\sum_{j=1}^{m_{i}} \mu\left(D_{j}^{i}\right) \geq \tilde{\mu}\left(w_{i}(B)\right) \geq p_{i} \mu(B) .
$$

Recall that $\left|D_{j}^{i}\right|<L_{i} r$ for $j=1, \ldots, m_{i}$. From the definition of the Lévy concentration function $Q_{\mu}$ it follows that

$$
\sum_{j=1}^{m_{i}} Q_{\mu}\left(L_{i} r\right) \geq p_{i} \mu(B) .
$$

Since $Q_{\mu}\left(L_{i} r\right)$ does not depend on the index $j$ we have

$$
m_{i} Q_{\mu}\left(L_{i} r\right) \geq p_{i} \mu(B) .
$$

The last inequality is true for every $B \subset X$ with $|B|<r$. Consequently,

$$
m_{i} Q_{\mu}\left(L_{i} r\right) \geq p_{i} Q_{\mu}(r) .
$$

Evidently this condition is equivalent to

$$
Q_{\mu}(r) \geq \frac{p_{i}}{m_{i}} Q_{\mu}\left(r / L_{i}\right) .
$$

Since $i \in I$ is arbitrary, it follows that

$$
Q_{\mu}(r) \geq \sup _{i \in I} \frac{p_{i}}{m_{i}} Q_{\mu}\left(r / L_{i}\right), \quad r \in\left(0, r_{0}\right] .
$$

According to Lemma 2 there exists $c>0$ such that

$$
Q_{\mu}(r) \geq c r^{s}, \quad r \in\left(0, r_{0}\right],
$$

where

$$
s=\min _{i \in I} \frac{\log \left(p_{i} / m_{i}\right)}{\log L_{i}} .
$$


Consequently,

$$
\overline{\operatorname{dim}}_{L} \mu \leq \min _{i \in I} \frac{\log \left(p_{i} / m_{i}\right)}{\log L_{i}}
$$

Theorem 1 immediately yields

COROllary 1. Let $d$ be the unique positive solution of the equation

$$
\sum_{i \in I} m_{i} L_{i}^{d}=1 .
$$

Put $p_{i}=m_{i} L_{i}^{d}$ for $i \in I$ and consider the IFS $\left\{\left(w_{i}, p_{i}\right): i \in I\right\}$. Suppose that a measure $\mu$ is invariant with respect to this system. Then

$$
\overline{\operatorname{dim}}_{L} \mu \leq d \text {. }
$$

In Theorem 1 we assume that all $L_{i}$ are strictly positive and this condition is essentially used in the proof. Now we will show that the statement of Theorem 1 remains true if some of the constants $L_{i}$ are zero.

Theorem 2. Let an $\operatorname{IFS}\left\{\left(w_{i}, p_{i}\right): i \in\{1, \ldots, N+M\}\right\}$ be given. Assume that there exist nonexpansive maps $P_{i}: X \rightarrow \mathbb{R}^{k_{i}}$ for $i=1, \ldots, N+M$, constants $L_{i} \in(0,1)$ for $i=1, \ldots, N$ and constants $c_{i} \geq 0$ for $i=1, \ldots$, $N+M$ such that

$$
\rho\left(w_{i}(x), w_{i}(y)\right) \leq \max \left\{L_{i} \rho(x, y), c_{i}\left\|P_{i}(x)-P_{i}(y)\right\|_{i}\right\}, \quad x, y \in X,
$$

for $i=1, \ldots, N$ and

$$
\rho\left(w_{i}(x), w_{i}(y)\right) \leq c_{i}\left\|P_{i}(x)-P_{i}(y)\right\|_{i}, \quad x, y \in X,
$$

for $i=N+1, \ldots, N+M$. Suppose that $\mu$ is an invariant measure with respect to the given system. Then

$$
\overline{\operatorname{dim}}_{L} \mu \leq \min \left\{\frac{\log \left(p_{1} / m_{1}\right)}{\log L_{1}}, \ldots, \frac{\log \left(p_{N} / m_{N}\right)}{\log L_{N}}, k_{N+1}, \ldots, k_{N+M}\right\} .
$$

Proof. We may assume that all coefficients $c_{i}$ for $i>N$ are positive integers. Fix an integer $n \geq 2$ and define $L_{i}=1 / n$ for $i=N+1, \ldots, N+M$. For given $c_{i}$ and $L_{i}=1 / n, i>N$, the integer number $m_{i}$, required by the Covering Property, is equal to $q_{i}\left(c_{i} n\right)^{k_{i}}$, where $q_{i}$ is a constant depending on the norm in $\mathbb{R}^{k_{i}}$. For example, if $\mathbb{R}^{k_{i}}$ is endowed with the supremum norm, then $q_{i}=1$. Observe that the completed IFS $\left\{\left(w_{i}, p_{i}\right): i \in\{1, \ldots, N+M\}\right\}$ has the Squeezing Property. Applying Theorem 1 we obtain

$$
\overline{\operatorname{dim}}_{L} \mu \leq \min \left\{\frac{\log \left(p_{1} / m_{1}\right)}{\log L_{i}}, \ldots, \frac{\log \left(p_{N+M} / m_{N+M}\right)}{\log L_{N+M}}\right\} .
$$

For $i>N$, we have

$$
\frac{\log \left(p_{i} / m_{i}\right)}{\log L_{i}}=\frac{\log \left(p_{i} q_{i}\right)-k_{i} \log n-k_{i} \log c_{i}}{-\log n} .
$$


Letting $n \rightarrow \infty$ we obtain

$$
\lim _{n \rightarrow \infty} \frac{\log \left(p_{i} / m_{i}\right)}{\log L_{i}}=k_{i} \quad \text { for } i=N+1, \ldots, N+M .
$$

From the last observation and inequality (10) the statement of Theorem 2 follows immediately.

4. Upper bound for thin dimension. Given $\mu \in \mathcal{M}_{1}(X)$ we define the lower and upper thin dimensions by the formulas

$$
\underline{\operatorname{dim}}_{T} \mu=\liminf _{r \rightarrow 0} \frac{\log T_{\mu}(r)}{\log r}, \quad \overline{\operatorname{dim}}_{T} \mu=\limsup _{r \rightarrow 0} \frac{\log T_{\mu}(r)}{\log r},
$$

where

$$
T_{\mu}(r)=\inf \{\mu(B(x, r)): x \in \operatorname{supp} \mu\} \quad \text { for } r>0 .
$$

The function $T_{\mu}:(0, \infty) \rightarrow[0,1]$ will be called the thin function corresponding to the measure $\mu$. Note that the values $T_{\mu}(r)$ are positive if $\operatorname{supp} \mu$ is a compact set. In general $T_{\mu}(r)$ is only nonnegative and we adopt the convention that $\log 0=-\infty$.

We are going to find upper bounds on the thin dimension of the invariant measure for so called condensation systems (see [1]). Let now $(X, \varrho)$ denote a complete, separable metric space.

Let $w_{i}: X \rightarrow X, i=1, \ldots, N$, be a sequence of functions satisfying the Lipschitz conditions

$$
\varrho\left(w_{i}(x), w_{i}(y)\right) \leq L_{i} \varrho(x, y) \quad \text { for } x, y \in X, i=1, \ldots, N .
$$

Let $C$ be an arbitrary finite subset of $X$ and let $w_{0}: X \rightarrow C$ be a given function. Further, let $\left(p_{0}, p_{1}, \ldots, p_{N}\right)$ be a probability vector, i.e.

$$
\sum_{i=0}^{N} p_{i}=1, \quad p_{k}>0 \quad \text { for } k=0, \ldots, N .
$$

Theorem 3. Suppose that the IFS $\left\{\left(w_{i}, p_{i}\right): i \in\{0, \ldots, N\}\right\}$ satisfies conditions (11) and (12). Assume that $w_{0}: X \rightarrow C$, where $C$ is a finite subset of $X$. Let $\mu$ be an invariant measure of the system $\left\{\left(w_{i}, p_{i}\right): i \in\{0, \ldots, N\}\right\}$, i.e.

$$
\mu(A)=\sum_{i=0}^{N} p_{i} \mu\left(w_{i}^{-1}(A)\right) \quad \text { for } A \in \mathcal{B}(X) .
$$

Further, assume that $\operatorname{supp} \mu$ is compact. Then

$$
\overline{\operatorname{dim}}_{T} \mu \leq \max _{1 \leq i \leq N} \frac{\log p_{i}}{\log L_{i}} .
$$


Proof. Standard calculation shows that for every measurable function $w: X \rightarrow X$ we have

$$
\operatorname{supp}\left(\mu \circ w^{-1}\right) \subset \overline{w(\operatorname{supp} \mu)} .
$$

From (13) it follows that

$$
\operatorname{supp} \mu=\bigcup_{i=0}^{N} \operatorname{supp}\left(\mu \circ w_{i}^{-1}\right),
$$

and by (14),

$$
\operatorname{supp} \mu \subset \operatorname{supp}\left(\mu \circ w_{0}^{-1}\right) \cup \overline{w_{1}(\operatorname{supp} \mu)} \cup \cdots \cup \overline{w_{N}(\operatorname{supp} \mu)} \text {. }
$$

Since $K=\operatorname{supp} \mu$ is compact, the last inclusion can be rewritten in the form

$$
K \subset \operatorname{supp}\left(\mu \circ w_{0}^{-1}\right) \cup w_{1}(K) \cup \cdots \cup w_{N}(K) .
$$

Fix $r_{0}>0$ and let $r \in\left(0, r_{0}\right)$. Since the function $X \ni x \mapsto \mu\left(B^{\circ}(x, r)\right)$ is lower semicontinuous, by the Weierstrass theorem there exists $\tilde{x} \in K$ such that

$$
\mu\left(B^{\circ}(\tilde{x}, r)\right)=\inf \left\{\mu\left(B^{\circ}(x, r)\right): x \in K\right\}
$$

Consequently,

$$
T_{\mu}(r)=\mu\left(B^{\circ}(\tilde{x}, r)\right) .
$$

By (15) the point $\tilde{x}$ belongs to $\bigcup_{i=1}^{N} w_{i}(K)$ or to $\operatorname{supp}\left(\mu \circ w_{0}^{-1}\right)$.

Suppose first that $\tilde{x} \in \bigcup_{i=1}^{N} w_{i}(K)$. This means that $\tilde{x}=w_{i}(y)$ for some $y \in K$. On the other hand, observe that

$$
w_{i}^{-1}\left(B^{\circ}(\tilde{x}, r)\right) \supset B^{\circ}\left(y, r / L_{i}\right) .
$$

Indeed, for $z \in B^{\circ}\left(y, r / L_{i}\right)$, we have

$$
\rho\left(\tilde{x}, w_{i}(z)\right)=\rho\left(w_{i}(y), w_{i}(z)\right)<L_{i} \rho(y, z)<r,
$$

which means that $w_{i}(z) \in B^{\circ}(\tilde{x}, r)$. By (13) and (16) we have

$$
\mu\left(B^{\circ}(\tilde{x}, r)\right) \geq p_{i} \mu\left(w_{i}^{-1}\left(B^{\circ}(\tilde{x}, r)\right)\right) \geq p_{i} \mu\left(B^{\circ}\left(y, r / L_{i}\right)\right) \geq p_{i} T_{\mu}\left(r / L_{i}\right) .
$$

Consequently,

$$
T_{\mu}(r) \geq p_{i} T_{\mu}\left(r / L_{i}\right) \quad \text { for } r \in\left(0, r_{0}\right] .
$$

Suppose now that $\tilde{x} \in \operatorname{supp}\left(\mu \circ w_{0}^{-1}\right)$. Then (14) yields $\operatorname{supp}\left(\mu \circ w_{0}^{-1}\right)$ $\subset \overline{w_{0}(K)}$. But $w_{0}(K)$ is a finite set. Consequently, so is $\operatorname{supp}\left(\mu \circ w_{0}^{-1}\right)$, say $\left\{c_{1}, \ldots, c_{m}\right\}$. Obviously $\left(\mu \circ w_{0}^{-1}\right)\left(\left\{c_{j}\right\}\right)>0$ for $j=1, \ldots, m$. Let

$$
\alpha_{\mu}=\min _{1 \leq j \leq m}\left(\mu \circ w_{0}^{-1}\right)\left(\left\{c_{j}\right\}\right) .
$$

Since $\tilde{x} \in\left\{c_{1}, \ldots, c_{m}\right\}$ we have

$$
\left(\mu \circ w_{0}^{-1}\right)\left(B^{\circ}(\tilde{x}, r)\right) \geq \alpha_{\mu} \quad \text { for } r \in\left(0, r_{0}\right],
$$


and by (13),

$$
\mu\left(B^{\circ}(\tilde{x}, r)\right) \geq p_{0}\left(\mu \circ w_{0}^{-1}\right)\left(B^{\circ}(\tilde{x}, r)\right) \geq p_{0} \alpha_{\mu} \quad \text { for } r \in\left(0, r_{0}\right] .
$$

Finally, from the last inequality and conditions (17) it follows that

$$
T_{\mu}(r) \geq \min \left\{p_{0} \alpha_{\mu}, p_{1} T_{\mu}\left(r / L_{1}\right), \ldots, p_{N} T_{\mu}\left(r / L_{N}\right)\right\}, \quad r \in\left(0, r_{0}\right] .
$$

The last inequality says that the function $\psi(r)=T_{\mu}(r)$ satisfies condition (7) with $p_{0} \alpha_{\mu}$ in place of $\alpha$.

Now consider the function

$$
\varphi(r)=c r^{s}, \quad \text { where } \quad 0<c \leq p_{0} \alpha_{\mu} \quad \text { and } \quad s \geq \max _{1 \leq i \leq N} \frac{\log p_{i}}{\log L_{i}} .
$$

It is easy to see that $\varphi$ satisfies condition (8).

Observe that the smallest value of $\psi$ in the interval $\left[\operatorname{lr}_{0}, r_{0}\right]$ is $T_{\mu}\left(l r_{0}\right)$. On the other hand, the largest value of $\varphi$ in $\left[l r_{0}, r_{0}\right]$ is $\varphi\left(r_{0}\right)=c r_{0}^{s}$. Thus, for a sufficiently small constant $c$, inequality (9) holds in $\left[l r_{0}, r_{0}\right]$, and by Lemma 3 this inequality holds for every $r \in\left(0, r_{0}\right]$.

Obviously

$$
\overline{\operatorname{dim}}_{T} \mu=\limsup _{r \rightarrow 0} \frac{\log T_{\mu}(r)}{\log r} \leq \limsup _{r \rightarrow 0} \frac{\log \varphi(r)}{\log r}=s .
$$

Hence the statement of Theorem 3 follows immediately.

The example below shows that the statement of Theorem 3 fails to hold without the assumption that $C$ is a finite subset of $X$.

Example 1. Consider the set

$$
X=[0,13 / 8] \cup\left\{a_{n}: n=0,1, \ldots\right\} \cup\{9 / 4\},
$$

where

$$
a_{0}=2 \quad \text { and } \quad a_{n}=2+\frac{3}{2 \pi^{2}} \sum_{k=1}^{n} \frac{1}{k^{2}} \quad \text { for } n \geq 1 .
$$

On the space $X$ consider the functions

$$
w_{1}(x)=\frac{1}{2} x, \quad w_{2}(x)=\frac{1}{2} x+\frac{1}{2} \quad \text { for } x \in X,
$$

and

$$
w_{0}(x)= \begin{cases}2 & \text { for } x \in[0,13 / 8] \\ a_{n} & \text { for } x=a_{n-1}, n=1,2, \ldots, \\ 9 / 4 & \text { for } x=9 / 4 .\end{cases}
$$

Since $\sum_{k=1}^{\infty}\left(1 / k^{2}\right)=\pi^{2} / 6$, the sequence $\left\{a_{n}\right\}$ converges to $9 / 4$. Obviously the functions $w_{1}$ and $w_{2}$ are similarities with scaling factor $L_{1}=L_{2}=1 / 2$ and $w_{0}$ is a Lipschitzian function with Lipschitz constant $L_{0}=1$. 
Observe that the IFS $\left\{\left(w_{i}, p_{i}\right): i=0,1,2\right\}$, where $p_{i}=1 / 3, i=0,1,2$, satisfies condition (5) and so it is asymptotically stable. Let $\mu_{*}$ be the corresponding invariant measure. Obviously, IFS $\left\{w_{i}: i=0,1,2\right\}$ is regular. Let $K$ be the corresponding semifractal. According to Proposition 2 we have $K=\operatorname{supp} \mu_{*}$. Clearly,

$$
K=w_{0}(K) \cup w_{1}(K) \cup w_{2}(K) .
$$

Set $D=\overline{\left\{a_{n}: n=0,1, \ldots\right\}}$. From (3) it follows easily that $D \subset K$. In addition we have

$$
w_{0}(K)=D, \quad w_{1}(K) \subset[0,9 / 8], \quad w_{2}(K) \subset[1 / 2,13 / 8] .
$$

Thus $w_{0}^{-1}\left(w_{1}(K)\right)=\emptyset$ and $w_{0}^{-1}\left(w_{2}(K)\right)=\emptyset$. Consequently,

$$
\left(\mu_{*} \circ w_{0}^{-1}\right)\left(w_{1}(K)\right)=0 \text { and }\left(\mu_{*} \circ w_{0}^{-1}\right)\left(w_{2}(K)\right)=0 .
$$

In particular, since $\operatorname{supp} \mu_{*}=K$ and $w_{0}^{-1}\left(w_{0}(K)\right) \supset K$ we have

$$
\left(\mu_{*} \circ w_{0}^{-1}\right)\left(w_{0}(K)\right)=1 .
$$

Using (4), (19) and the last equality we obtain

$$
\begin{aligned}
\mu_{*}\left(w_{0}(K)\right) & =\frac{1}{3} \mu_{*}\left(w_{0}^{-1}\left(w_{0}(K)\right)\right)+\frac{1}{3} \mu_{*}\left(w_{1}^{-1}\left(w_{0}(K)\right)\right)+\frac{1}{3} \mu_{*}\left(w_{2}^{-1}\left(w_{0}(K)\right)\right) \\
& =\frac{1}{3} .
\end{aligned}
$$

Observe also that for $n \geq 2$,

$$
w_{0}^{n}(K)=\overline{\left\{a_{n-1}, a_{n}, \ldots\right\}}
$$

and

$$
w_{0}^{-1}\left(w_{0}^{n}(K)\right)=\overline{\left\{a_{n-2}, a_{n-1}, \ldots\right\}}=w_{0}^{n-1}(K) .
$$

By an induction argument it is easy to verify that

$$
\begin{aligned}
\mu_{*}\left(w_{0}^{n}(K)\right) & =\frac{1}{3} \mu_{*}\left(w_{0}^{-1}\left(w_{0}^{n}(K)\right)\right)+\frac{1}{3} \mu_{*}\left(w_{1}^{-1}\left(w_{0}^{n}(K)\right)\right)+\frac{1}{3} \mu_{*}\left(w_{2}^{-1}\left(w_{0}^{n}(K)\right)\right) \\
& =\frac{1}{3} \mu_{*}\left(w_{0}^{-1}\left(w_{0}^{n}(K)\right)\right)=\frac{1}{3^{n}} .
\end{aligned}
$$

Thus

$$
\begin{aligned}
\mu_{*}\left(\left\{a_{n}\right\}\right) & =\mu_{*}\left(w_{0}^{n+1}(K) \backslash w_{0}^{n+2}(K)\right) \\
& =\mu_{*}\left(w_{0}^{n+1}(K)\right)-\mu_{*}\left(w_{0}^{n+2}(K)\right) \\
& =1 / 3^{n+1}-1 / 3^{n+2}=2 / 3^{n+2} .
\end{aligned}
$$

Finally, observe that for $r_{n}=3 /\left(4 \pi^{2} n^{2}\right)$ we have $B^{\circ}\left(a_{n}, r_{n}\right)=\left\{a_{n}\right\}$. Consequently,

$$
T_{\mu_{*}}\left(r_{n}\right) \leq \mu_{*}\left(\left\{a_{n}\right\}\right) \leq 2 / 3^{n+2} .
$$


It follows that

$$
\frac{\log T_{\mu_{*}}\left(r_{n}\right)}{\log r_{n}} \geq \frac{\log \left(2 / 3^{n+2}\right)}{\log r_{n}}=\frac{\log 2-(n+2) \log 3}{\log \left(3 / 4 \pi^{2}\right)-2 \log n} .
$$

Letting $n \rightarrow \infty$ we obtain

$$
\underline{\operatorname{dim}}_{T} \mu_{*}=\infty
$$

\section{References}

[1] M. F. Barnsley, Fractals Everywhere, Academic Press, New York, 1988.

[2] F. Bloom and W. Hao, The $L^{2}$ squeezing property for nonlinear bipolar viscous fluids, J. Dynam. Differential Equations 6 (1994), 513-542.

[3] I. Chueshov and S. Siegmund, On dimension and metric properties of trajectory attractors, ibid. 17 (2005), 621-641.

[4] L. Dung and B. Nicolaenko, Exponential attractors in Banach spaces, J. Differential Equations 13 (2001), 791-806.

[5] A. Eden, C. Foias, B. Nicolaenko and R. Temam, Exponential Attractors for Dissipative Evolution Equation, Masson, Paris, 1994.

[6] M. Efendiev, A. Miranville and S. Zelik, Exponential attractors for a nonlinear reaction-diffusion system in $\mathbb{R}^{3}$, C. R. Acad. Sci. Paris Sér. I 330 (2000), 713-718.

[7] H. Gacki, Applications of the Kantorovich-Rubinstein maximum principle in the theory of Markov semigroups, Dissertationes Math. 448 (2007), 59 pp.

[8] J. Jaroszewska and M. Rams, On the Hausdorff dimension of invariant measures of weakly contracting on average measurable IFS, J. Statist. Phys. 132 (2008), 907-919.

[9] T. Jordan and M. Pollicott, The Hausdorff dimension of measures for iterated function systems which contract on average, Discrete Contin. Dynam. Systems 22 (2008), $235-246$.

[10] A. J. Koksch and J. Norbert, A modified strong squeezing property and inertial manifolds of semiflows, Arch. Math. (Brno) 36 (2000), 477-486.

[11] A. Lasota, A variational principle for fractal dimension, J. Nonlinear Anal. 64 (2006), 618-628

[12] A. Lasota and J. Myjak, Semifractals on Polish spaces, Bull. Polish Acad. Sci. Math. 46 (1998), 179-196.

[13] —, - On a dimension of measures, ibid. 50 (2002), 221-235.

[14] A. Lasota and J. Traple, Dimension of invariant sets for mappings with the squeezing property, Chaos Solitons Fractals 28 (2006), 1271-1280.

[15] J. Mallet-Paret, Negatively invariant sets of compact maps and an extension of a theorem of Cartwright, J. Differential Equations 22 (1976), 331-341.

[16] P. A. P. Moran, Additive functions on intervals and Hausdorff measure, Proc. Cambridge Philos. Soc. 42 (1946), 15-23.

[17] D. Pražak, On finite fractal dimension of the global attractor for the wave equation with nonlinear damping, J. Differential Equations 14 (2002), 763-776.

[18] K. S. Promislov, The squeezing property and the decay of small vavelength in dissipative dynamical systems, Appl. Anal. 53 (1994), 233-239.

[19] M. Rams, Contracting-on-average baker maps, Bull. Polish Acad. Sci. Math. 54 (2006), 219-229. 
[20] T. Szarek, The dimension of self-similar measures, ibid. 48 (2000), 293-302.

H. Gacki

Institute of Mathematics

Silesian University

Bankowa 14

40-007 Katowice, Poland

E-mail: henryk.gacki@us.edu.pl
J. Myjak Dipartimento di Matematica Pura ed Applicata Università di L'Aquila Via Vetoio 67100 L'Aquila, Italy E-mail: myjak@univaq.it and

WMS AGH

Al. Mickiewicza 30 30-059 Kraków, Poland E-mail: myjakjoz@mat.agh.edu.pl

Received March 17, 2009;

received in final form August 10, 2009 